\title{
Cricoarytenoid Muscle
}

National Cancer Institute

\section{Source}

National Cancer Institute. Cricoarytenoid Muscle. NCI Thesaurus. Code C32398.

A pair of muscles, the lateral cricoarytenoid and the posterior cricoarytenoid, that connect the cricoid cartilage and arytenoid cartilage. The posterior cricoarytenoid is the sole abductor of the vocal folds. The lateral cricoarytenoid is the main antagonist of the posterior cricoarytenoid. 